\title{
Research on Small Hydropower Group Output Characteristics of Multiple Time Scales
}

\author{
Xiankui Wen a , Li Su, Chenghui Lin, Qiang Fan, Jiqun Tang, Cheng Mao \\ Guizhou power grid co., LTD electric power research institute, Guizhou Guiyang, 550002 \\ a13985410224@139.com
}

\begin{abstract}
Most of the small hydropower has no power regulation capacity, which affects the operation of the power grid. By analyzing the average daily output characteristics of a single small hydropower station, it found its power curve quite different. The small hydropower in the similar geographical environment is classified as a cluster. Analysis of power generation characteristics of small hydropower station at different time scales, the output of small hydropower in each area has obvious characteristics of Wet-Dry, strong correlation of output, but with the expansion of the scope of the space gradually weakened. With the shortening of time scale, the output fluctuation of small hydropower stations in the adjacent time section is gradually reduced, so it provides reference for the analysis of small hydropower generation operation.
\end{abstract}

Keywords: Mountain area; Small hydropower group; Multi-time scale; Output characteristics.

\section{Introduction}

China is one of the countries with the most abundant hydropower resources in the world. In recent years, small hydropower has been vigorously developed, with its advantages of less investment, short development period, no pollution, and has made an important contribution to China's energy problems. Small hydro generating units greatly meet the use of electricity in rural areas, promote the local economic development, and to achieve rural electrification, economic development and improve the level of social life have a very important significance.[1] Most small hydropower stations are runoff type hydropower station, and have no regulatory capacity. The running state and the output of most small hydropower stations are completely controlled by the rainfall, which have lag effect and the accumulation effect of uncertainty $[2,3]$. Small hydropower station power output fluctuations frequently, and great impact on the power grid, which make the law of power grid load is difficult to grasp [4].

\section{Small hydropower in Guizhou area}

Guizhou is rich in water resources, and has a large number of small hydropower. As of 2015, there are 1286 small hydropower stations in Guizhou power grid, the installed capacity of about 3600MW. There are 847 power plants installed capacity of less than $1000 \mathrm{~kW}$, accounting for $68 \%$, and 570 power

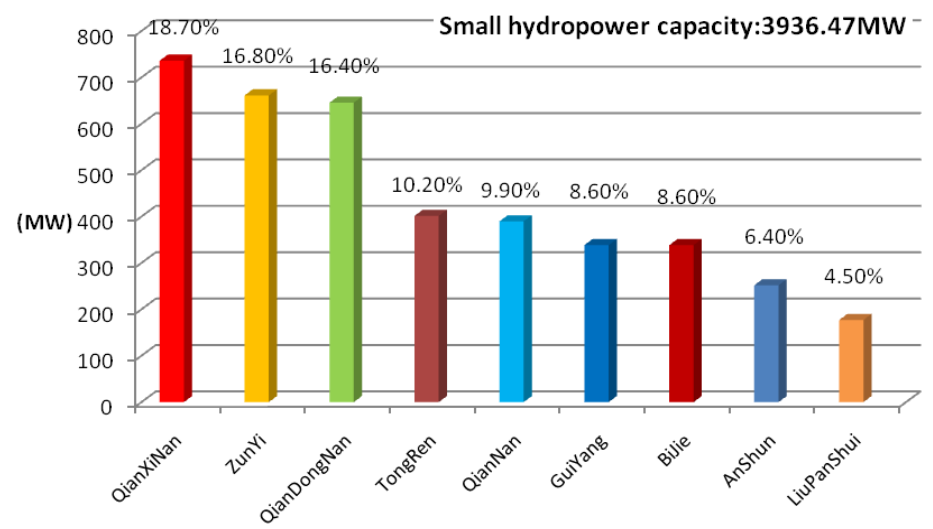

Fig. 1 Small hydropower capacity in Guizhou in 2015 
Plants installed capacity of less than 500kW, accounting for $46 \%$ [5].Figure 1 is the province's various regions of the small hydropower installed statistics. Most of the small hydropower stations in Guizhou are runoff type, and no regulatory capacity. In actual operation, the running state and output of small hydropower is completely controlled by rainfall, and with the rainfall seasons will appear in wet and dry seasons.

\section{Select the research sample}

Due to the complex geographical environment and changeable weather in Guizhou mountainous area, the distribution of rainfall in the same area is also very uneven. So the output of single small hydropower in different position is different, volatile and intermittent, which makes research of single small hydropower is a difficult and does not have the universal significance. The geographical environment is similar to a number of small hydropower is classified as a cluster, because of its geographical environment and meteorological factors have a certain similarity, the output trend of the small hydropower is more consistent. This paper choose the Maojiadong, Moshi and Zhongyuke small hydropower stations constitute the small hydropower clusters, and draw between clusters and small hydropower output correlation graph is shown in Figure 2.

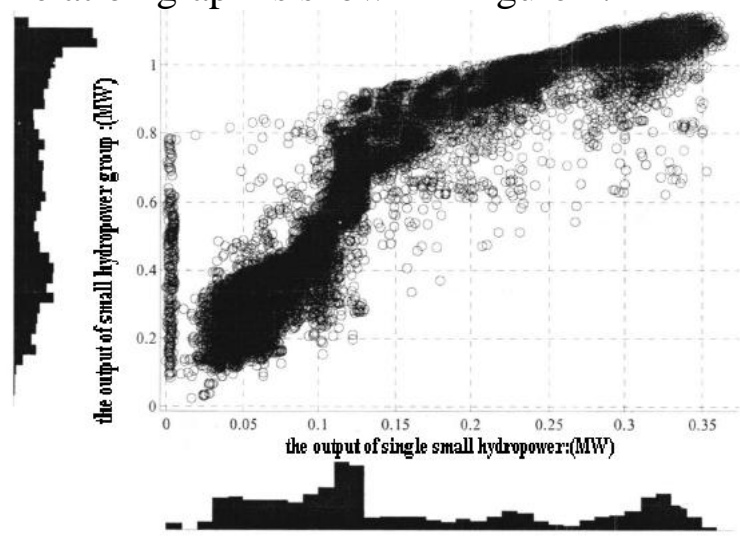

Fig. 2 the output correlation between a single small hydropower and small hydropower group

\section{Long term (monthly) output characteristics of small hydropower station}

The monthly average power and the monthly correlation coefficient of the small hydropower station are analyzed by the long term (monthly) fluctuation of small hydropower station. Figure 3 is the province, Bijie area and Liu panshui area of the average power curve of each month between October 2015 and November 2014. From June to October, small hydropower is maintained at a relatively high level of output, but from January to April the output of small hydropower can only reach about $30 \%$.

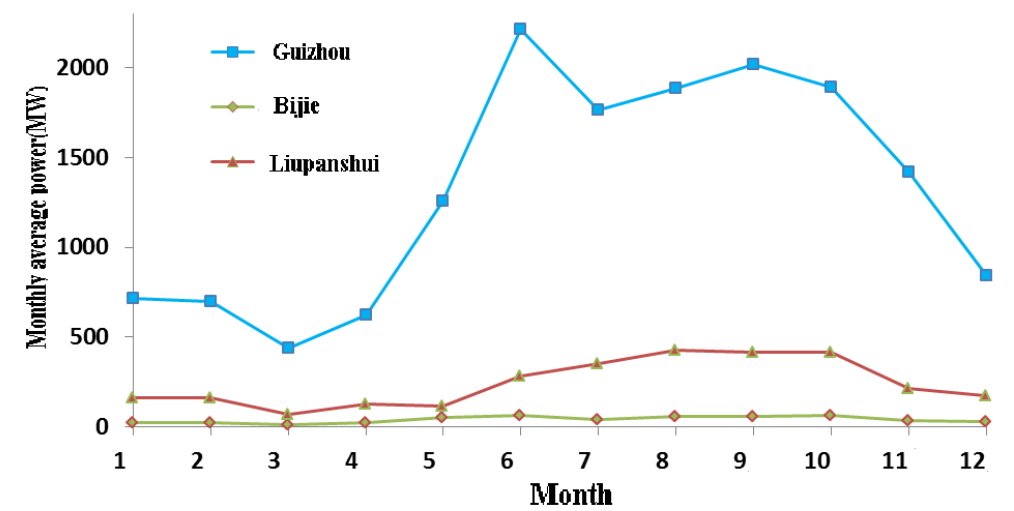

Fig. 3 the Small hydropower monthly power curve of BiJie, Liu PanShui, and GuiZhou

Table 1 is monthly correlation coefficient of regional output in Guizhou based on the calculation of the monthly average output of the small hydropower station. 
Table 1 Monthly correlation coefficient of regional output in Guizhou

\begin{tabular}{|c|c|c|c|c|c|c|c|c|c|c|}
\hline & GuiYang & AnShun & BiJie & DuYun & KaiLi & LiuPanShui & TongRen & XingYi & ZunYi & GuiZhou \\
\hline GuiYang & 1.00 & 0.84 & 0.88 & 0.88 & 0.51 & 0.80 & 0.79 & 0.93 & 0.87 & 0.96 \\
\hline AnShun & & 1.00 & 0.82 & 0.77 & 0.64 & 0.84 & 0.66 & 0.95 & 0.85 & 0.91 \\
\hline BiJie & & & 1.00 & 0.92 & 0.58 & 0.80 & 0.79 & 0.86 & 0.86 & 0.94 \\
\hline DuYun & & & & 1.00 & 0.60 & 0.66 & 0.93 & 0.79 & 0.93 & 0.94 \\
\hline KaiLi & & & & & 1.00 & 0.37 & 0.62 & 0.58 & 0.62 & 0.62 \\
\hline LiuPanShui & & & & & & 1.00 & 0.42 & 0.88 & 0.63 & 0.85 \\
\hline TongRen & & & & & & & 1.00 & 0.64 & 0.94 & 0.82 \\
\hline XingYi & & & & & & & & 1.00 & 0.81 & 0.94 \\
\hline ZunYi & & & & & & & & & 1.00 & 0.91 \\
\hline GuiZhou & & & & & & & & & & 1.00 \\
\hline
\end{tabular}

The various regions and the province's small hydropower correlation coefficient is between 0.62-0.96, and the correlation coefficient between the small hydro powers in each region is between 0.37 and 0.95 . The smallest correlation coefficient is between Liu panshui and Kaili, as the distance between the two places is far.

\section{Short term output characteristics of small hydropower station}

\subsection{Daily output fluctuation characteristic}

Daily output fluctuation characteristic of small hydropower station is described by the peak valley ratio within one day. The peak valley ratio within one day refers to the maximum output of small hydropower group minus minimum output divided by the installed capacity. Figure 4 is a frequency chart of peak valley ratio of small hydropower group in the Dafang County, Hezhang County and Bijie area from May 2012 to April 2013.The small hydropower group in Dafang County connected to the Piaojing substation, including: Dashan power station, Loubu power station, Changshiche power station, etc. 10 small hydropower. The small hydropower group in Hezhang County connected to the Longquan substation, including: Zhongyuke power station, Kashang power station, Xieshui power station, etc.

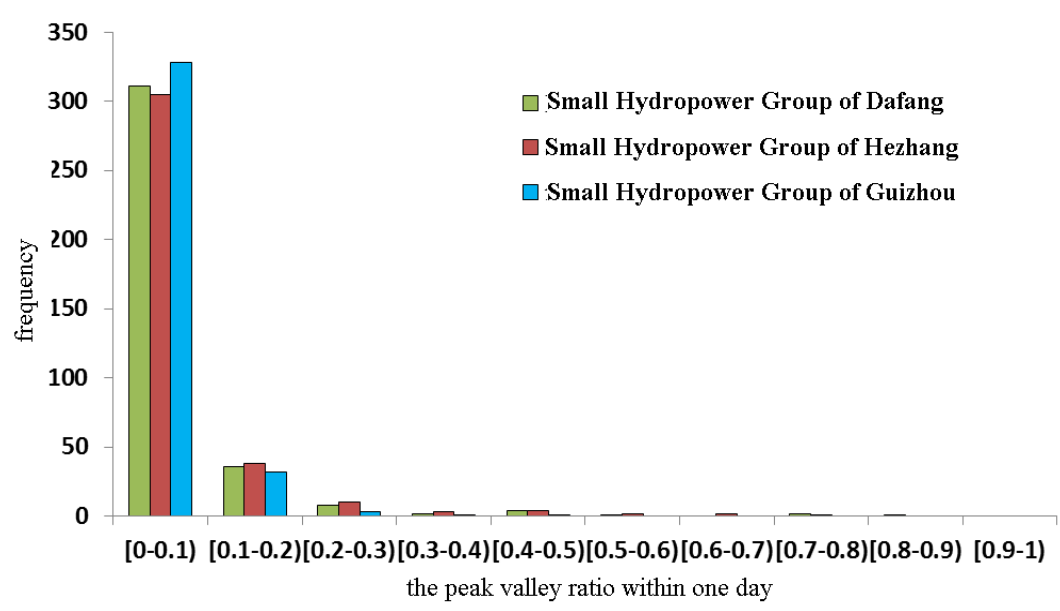

Fig. 4 a frequency chart of peak valley ratio of small hydropower group

Data show that the daily peak valley ratio of three small hydropower group is concentrated in between 0 to 0.2 . The small hydropower group of Dafang County, the small hydropower group of Hezhang County and the small hydropower group of Bijie area, in contrast of the daily peak valley ratio is less than 0.2 percentage accounted for $95.07 \%, 93.97 \%$ and $98.63 \%$. Therefore, the daily peak valley ratio values of three small hydropower groups greater than 0.2 accounted for only a very small proportion. It shows that the change of the daily output of the small hydropower station is small, and it is basically maintained in a stable output state, only when the sudden and continuous rainfall can make the daily output change dramatically.

\subsection{Hour output fluctuation characteristic}

Hour output fluctuation characteristic of small hydropower station is described by the fluctuation ratio. The fluctuation ratio is the difference between the small hydropower groups of adjacent 
moment output divided by the installed capacity. Figure 5 is the probability distribution graph of hourly fluctuation ratio of Hezhang small hydropower group during the period from April 2013 to May 2012. The 0 points of vertical coordinate in the graph show the probability statistics which the fluctuation ratio fall into the $[-0.5,+0.5]$. By analysis, fluctuation ratio in $[-0.5 \%,+0.5 \%]$ within the scope of the probability for $39.23 \%$, fluctuation ratio in $[-5 \%,+5 \%]$ within the scope of the probability of $96.30 \%$, in [-10\%, $+10 \%$ ] range probability is increased is $98.07 \%$, the absolute maximum fluctuation ratio is about $32 \%$. The small hydropower group output is less volatility in hours.

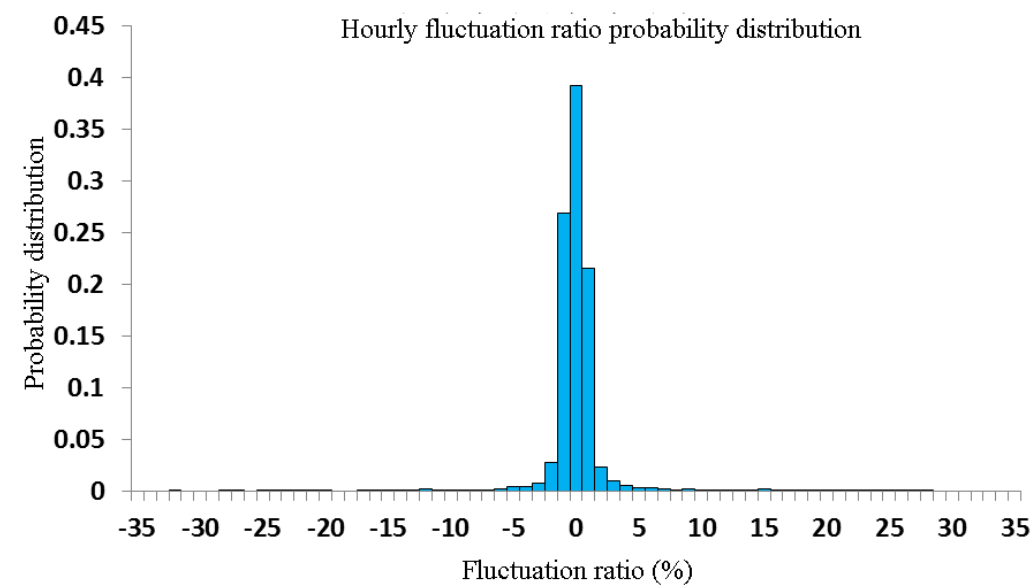

Fig. 5 fluctuation ratio probability distribution of small hydropower output in hour

\section{Conclusion}

The output of single small hydropower has a large difference, so the research of single small hydropower is very difficult. The small hydropower group with similar geographical and meteorological factors has a strong correlation, and it is a good practical value to study it in a cluster. In terms of long-term output characteristics, the monthly average power distribution of small hydropower in the province is very uneven, showing obvious characteristics of the abundance and dry. The geographical environment and climate factors in different regions of the small hydropower group were significantly different, and the output consistency of each small hydropower stations is relatively dispersed. The output correlation of small hydropower group's in a relatively recent area is stronger than that in a distant area. In terms of short-term output characteristics, the output of small hydropower group is no significant change, and the output is basically maintained at a steady state. With the reduction of time scale, the output fluctuation of small hydropower group in the adjacent time section is gradually reduced, and only the sudden and continuous rainfall will make the daily output change dramatically.

\section{Acknowledgements}

This work was financially supported by the national science and technology support project (2013BAA02B02) \& Guizhou province science and technology major project ([2014]6022).

\section{References}

[1]. General report of the survey and evaluation results (2008) of the rural water energy resources of the People's Republic of China Excerpts-Water resources and its distribution in rural areas [J]. China Water Resources. Vol.10 (2009), p. 10-14

[2]. Xu Wei, Luo Xin, Liu Mei. Application of Two-Phase Reduction Method in Load Forecasting for Regions with Abundant Small Hydropower [J], power system technology .Vol.33 (2009), p. $87-92$ 
[3]. Li Wei, Sun Dian. The Weather Factor in Short-term Load Forecast of Hubei Grid and the Strategy for the Influence of Small Hydraulic Power Plant [J]. Hubei electric power, Vol.35 (2011), p. 6-8

[4]. Li Shiwei, Ge Minwu, Jin Yubin. Analysis of the impact of small hydropower stations on the power grid [J]. China's rural water conservancy electric power, Vol8 (2012), p. 152-154

[5]. Gu Yaonan. Study on the model of small hydropower purchase in Guizhou Power Grid Corp [D]. Guizhou University, 2015. 